\title{
Development of Ray Tracing Algorithms for Scanning Plane and Transverse Plane Analysis for Satellite Multibeam Application
}

\author{
N. H. Abd Rahman, ${ }^{1,2}$ M. T. Islam, ${ }^{1}$ N. Misran, ${ }^{1}$ Y. Yamada, ${ }^{3}$ and N. Michishita ${ }^{3}$ \\ ${ }^{1}$ Department of Electrical, Electronic and Systems Engineering, Faculty of Engineering and Built Environment, \\ Universiti Kebangsaan Malaysia (UKM), 43600 Bangi, Malaysia \\ ${ }^{2}$ Faculty of Electrical Engineering, Universiti Teknologi MARA, 40450 Shah Alam, Selangor, Malaysia \\ ${ }^{3}$ National Defense Academy, 1-10-20 Hashirimizu, Yokosuka 239-8686, Japan
}

Correspondence should be addressed to N. H. Abd Rahman; huda2811@gmail.com

Received 18 November 2013; Accepted 18 March 2014; Published 28 April 2014

Academic Editor: Rezaul Azim

Copyright ( 2014 N. H. Abd Rahman et al. This is an open access article distributed under the Creative Commons Attribution License, which permits unrestricted use, distribution, and reproduction in any medium, provided the original work is properly cited.

\begin{abstract}
Reflector antennas have been widely used in many areas. In the implementation of parabolic reflector antenna for broadcasting satellite applications, it is essential for the spacecraft antenna to provide precise contoured beam to effectively serve the required region. For this purpose, combinations of more than one beam are required. Therefore, a tool utilizing ray tracing method is developed to calculate precise off-axis beams for multibeam antenna system. In the multibeam system, each beam will be fed from different feed positions to allow the main beam to be radiated at the exact direction on the coverage area. Thus, detailed study on caustics of a parabolic reflector antenna is performed and presented in this paper, which is to investigate the behaviour of the rays and its relation to various antenna parameters. In order to produce accurate data for the analysis, the caustic behaviours are investigated in two distinctive modes: scanning plane and transverse plane. This paper presents the detailed discussions on the derivation of the ray tracing algorithms, the establishment of the equations of caustic loci, and the verification of the method through calculation of radiation pattern.
\end{abstract}

\section{Introduction}

Advances in wireless communications have introduced tremendous demands in the antenna technology $[1,2]$. Due to the significant raise in the number of geostationary satellites that provide high data rate services, the demands for satellite antenna system with multiple spot beams are also increasing. In satellite broadcasting application, fine contoured beam design is very critical to ensure uniform gain to the desired area and to decrease the radiation level in the unwanted areas rapidly. Thus, to produce these contoured beams, combination of more than one beam is employed [3-5]. Antenna structures for various multibeam scenarios have been discussed in [6].

Many reflector shaping methods and techniques have been identified as suitable to solve this issue. For example, in [7], physical optics (PO) technique has been employed to generate contoured beam from a shaped reflector antennas.
The shaping of the reflector is carried out by using third party commercial software called TICRA POS. To fulfill the high gain requirement, the feed is optimized and displaced laterally in the focal plane; however, the detailed analysis on how the lateral feed displacement relates to the beam shift is not performed.

Apart from the PO methods, many studies on the focal region of parabolic reflectors have been conducted. Previously, a reflector shaping technique based on the aperture diffraction method has been studied [8]. The technique has been employed in [9] to estimate feed positions for two distinctive surfaces, resulting in the best feed location for elevation and azimuth plane patterns. Based on the scanning of two planes, the best focal surfaces which are expressed in two-dimensional coordinate have been used to determine the antenna feed positions. However, the relationship of the caustic displacement to the parameter $f / D$ is not clear. 
In this paper, a design tool called ray tracing method is developed. The tool is designed to measure the caustics of a single parabolic reflector antenna system when radiated from various incident beam directions. Therefore, the same concept is used to determine the best feed positions for the satellite multibeam system. In this method, caustic points are determined based on the focal region generated by the convergence of rays observed in the scanning plane and transverse plane of the antenna system. The scanning plane and transverse plane analysis have been carried out previously for dielectric lens antenna [10] and the method has been very useful in determining feed positions. The calculation of rays and parabolic surface points are performed on the basis of mathematical and physical optics algorithms. To investigate the behaviour of caustics, the ray tracing program is performed for various antenna configurations and beam directions. The results are recorded and the data are interpolated. Based on the data, a set of equations to represent the loci of caustics are derived. These equations are very useful to determine the best feed position, especially for multibeam application. The reliability and accuracy of the equations are verified in this paper.

\section{Ray Tracing Concept}

2.1. Antenna and Feed Type. The antenna system comprises of one large parabolic reflector and one feeding element. The fundamental parabolic reflector configuration and the coordinate system are shown in Figure 1. The reflector is symmetrical around the $z$-axis, while $x$-axis and $y$-axis indicate the radial direction of the reflector. The origin of the coordinate system is expressed by $O$. Under a focused operation, a feed antenna is placed at the focal point, $O$, with distance $f$ away from the reflector.

During operation, rays emitted from the feed will reach the parabolic surface at a point on reflector surface, denoted by $P_{\theta \phi}$ that has vector components of $P_{x}, P_{y}, P_{z} . \phi$ corresponds to the angle around the $z$-axis and $\theta$ indicates the angle from the feed point to the $P_{\theta \phi}$. The calculation of reflector, in $x$-and $z$-coordinate component, is based on the standard equation of a parabolic surface [11]:

$$
z=\frac{x^{2}}{4 f}-f .
$$

The distance from the feed to the reflector surface, $\rho$, can be expressed in polar coordinates as follows [12]:

$$
\rho=\frac{2 f}{1+\cos \theta} .
$$

2.2. Design Flow. The flowchart expression of the developed ray tracing program is shown in Figure 2. The program is developed on MATLAB simulation tool. Both scanning plane and transverse plane have the same fundamental concept; however, the calculation algorithms are different, due to the variation in plane wave configurations.

The analysis is performed based on the receiving mode condition as explained in [13]. Initially, antenna parameters

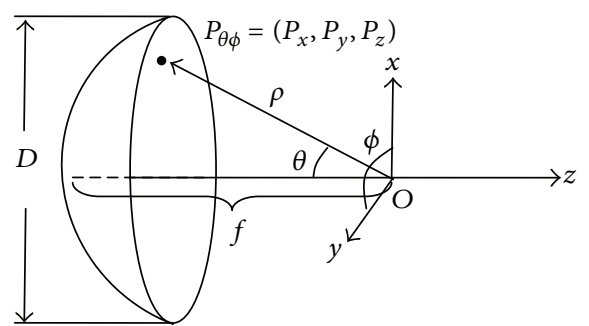

Figure 1: Antenna configuration.

such as focal length $(f)$, diameter $(D)$, and frequency are determined based on the spacecraft requirements and limitations. Other parameters such as reflector angle $(\theta)$ and distance to reflector $\operatorname{rim}(\rho)$ are calculated on the basis of mathematical equations, physical optic (PO) conditions, and trigonometry equations explained in the next section. In the focal region ray tracing, all of the incident rays coming from the desired beams will produce multiple reflections at the reflector surface. The intersection of the rays will converge on a plane or point and will form a set of traces and curves that are observed and studied in this paper. The convergence point or plane is called a caustic $[8,12]$. This position becomes the best feed point for the beam scanning angle $\theta_{\text {in }}$. The process is repeated for many sets of incident angles and antenna configurations.

\section{Ray Tracing Algorithm: Overview and Parametric Setup}

3.1. Receiving Mode Ray Tracing. The ray tracing algorithms are derived based on the receiving mode condition illustrated in Figure 3. The illumination is in a form of plane wave, transmitted from a fixed direction and tilted with respect to the reflector axis.

3.2. Scanning Plane. The illustration of scanning plane in a single reflector system is shown in Figure 4. For the calculation of caustic, the incident plane is displaced from the axis perpendicular to the reflector by an angle $\theta_{\text {in }}$ and the caustic position is measured. The incident rays exist in a vertical line that is contained in the $x z$-plane.

In the scanning plane, the reflector points, $P(\theta)$, will be determined in terms of $\rho$ and $\theta$, where $\rho$ is the distance measured from feed point $(F)$ to the reflector surface and $\theta$ is the angle from the origin to the reflector surface. To begin with, the $x$-coordinate of reflector surface $\left(P_{r x}\right)$ is set between the range of its minimum and maximum, $x_{1}=(-D / 2)$ to $(D / 2)$. By solving the simultaneous equations of (2) and its relation to $\theta$ from Figure 4, the expression of $\theta$ can be obtained as follows:

$$
\theta=\cos ^{-1}\left(\frac{4 f^{2}-x_{1}^{2}}{4 f^{2}+x_{1}^{2}}\right)
$$




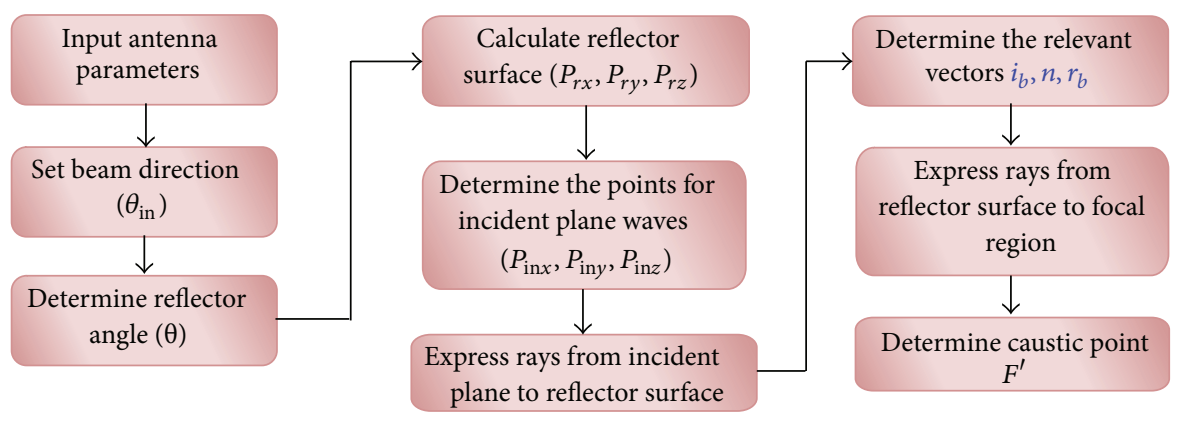

FIGURE 2: Flow chart of focal region ray tracing program.

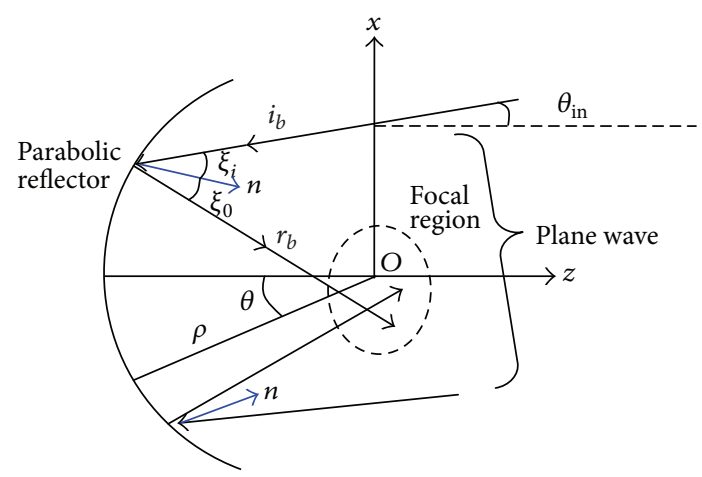

FIGURE 3: Illustration of focal region in the radiation mode ray tracing.

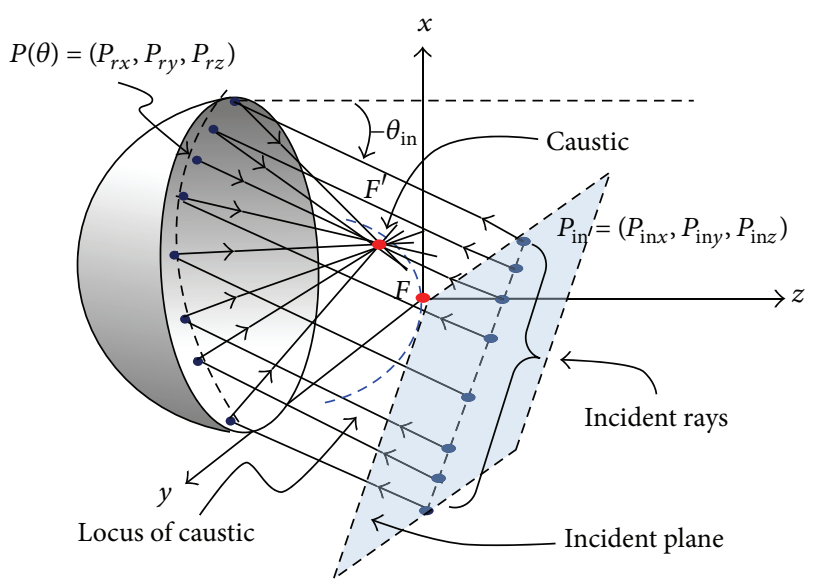

FIGURE 4: Illustration of scanning plane in a single reflector system.

Thus, by taking into account (3) and (1), the coordinate of $P(\theta)$ in $x$-, $y$-, and $z$-direction is defined as follows:

$$
\begin{gathered}
P_{r x}=x_{1}, \\
P_{r y}=0, \\
P_{r z}=\frac{\rho \sin ^{2} \theta}{4 f}-f .
\end{gathered}
$$

The incident point, known as $P_{\text {in }}\left(P_{\text {inx }}, P_{\text {in } y}, P_{\text {inz }}\right)$, is calculated based on the incident plane wave directed to the reflector with $\theta_{\text {in }}$ offset from the centre position. Thus, in general, by considering the incident beam, the $x$ - and $z$ component of incident plane can be expressed in tangent equation, as follows:

$$
\frac{\sin \theta_{\text {in }}}{\cos \theta_{\text {in }}}=\frac{x_{r}-x_{\text {in }}}{z_{r}-z_{\text {in }}}=\tan \theta_{\text {in }} .
$$

The line equation of the incident plane, $x_{\text {in }}$, where $l_{o}$ represents the length of the feed point, $F$, to the incident point, is generally expressed as follows:

$$
x_{\text {in }}=-\cot \theta_{\text {in }}\left(z_{\text {in }}-\frac{l_{o}}{\cos \theta_{\text {in }}}\right) .
$$

Therefore, by solving the simultaneous equations of (5) and (6), the coordinate of $P_{\text {in }}$ can be calculated as follows:

$$
\begin{gathered}
P_{\mathrm{in} x}=x_{\mathrm{in}}, \\
P_{\mathrm{in} y}=0, \\
P_{\mathrm{in} z}=P_{r z} \sin ^{2} \theta_{\mathrm{in}}-P_{r x} \sin \theta_{\mathrm{in}} \cos \theta_{\mathrm{in}}+l_{o} \cos \theta_{\mathrm{in}} .
\end{gathered}
$$

For the scanning plane analysis, the coordinate $P_{\text {iny }}$ remains zero as only the $\theta_{\text {in }}$ direction is taken into account and only vertical scanning is performed. Then, the unit incident vector, $i_{b}$, from a known beam direction $\left(P_{\text {in }}\right)$ to reflector surface $\left(P_{r}\right)$ is expressed by the following equation, where $i_{b x}, i_{b y}$, and $i_{b z}$ indicate the $x, y$, and $z$ components of the vector $i_{b}$ :

$$
\begin{aligned}
i_{b}\left(i_{b x}, i_{b y}, i_{b z}\right) & =\frac{\overrightarrow{P_{\text {in }} P_{r}}}{\left|\overrightarrow{P_{\text {in }} \mid}\right|} \\
& =\frac{\left[P_{\text {in } x}-P_{r x}, P_{\text {in } y}-P_{r y}, P_{\text {in } z}-P_{r z}\right]}{\left|\overrightarrow{P_{\text {in }} P_{r}}\right|} .
\end{aligned}
$$

The next step is to calculate the unit reflected vector, $r_{b}=$ $\left[r_{b x}, r_{b y}, r_{b z}\right]$. To solve that, firstly, the normal unit vector on the parabolic reflector, $n_{b}=\left[n_{b x}, n_{b y}, n_{b z}\right]$, is derived as follows:

$$
n_{b}=\left(-\sin \left(\frac{\theta}{2}\right), 0, \cos \left(\frac{\theta}{2}\right)\right)
$$




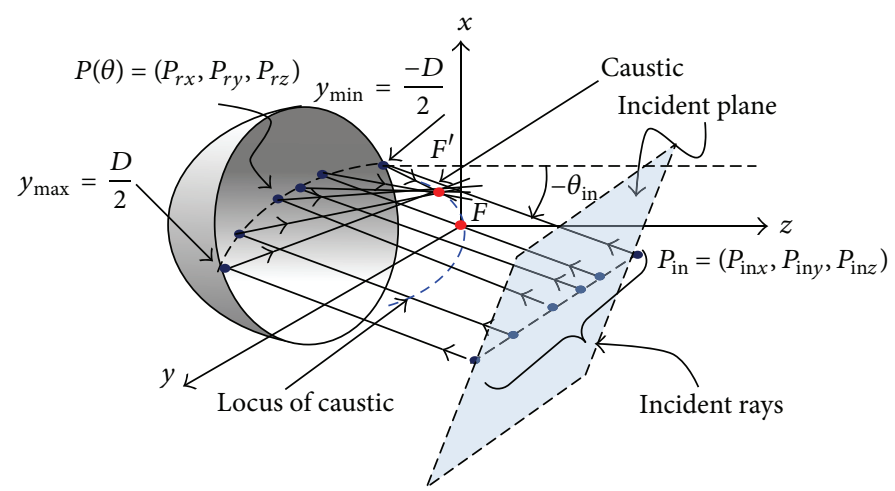

FIGURE 5: Illustration of transverse plane in a single reflector system.

Then, by applying the condition that all vectors exist on the same plane, the simultaneous equations of the vector dot product and cross product are solved [7]. The vectors $i_{b}$ and $n_{b}$ are expressed in terms of $\xi_{0}$ as follows, where $\xi_{0}$ represents the incident angle at the reflector surface:

$$
n_{b x} i_{b x}+n_{b y} i_{b y}+n_{b z} i_{b z}=\cos \xi_{0}
$$

The equation is expanded as follows, assuming that the incidence angle $\xi_{0}$ is equal to the reflected angle $\xi_{i}$ measured from the normal vector, $n_{b}$ :

$$
\begin{aligned}
& n_{b y} i_{b z}-n_{b z} i_{b y}=n_{b y} r_{b z}-n_{b z} r_{b y}, \\
& n_{b z} i_{b x}-n_{b x} i_{b z}=n_{b z} r_{b z}-n_{b x} r_{b y}, \\
& n_{b x} i_{b y}-n_{b y} i_{b x}=n_{b x} r_{b y}-n_{b y} r_{b x} .
\end{aligned}
$$

The left hand side of (11) is given by known values; therefore the equations become constant and are expressed in terms of $a_{b x}, a_{b y}$, and $a_{b z}$, respectively. Finally, by taking into account all the relationships defined above, the unknowns of $r_{b x}, r_{b y}$, and $r_{b z}$ are solved:

$$
\begin{aligned}
& r_{b x}=-n_{x} \cos \xi_{0}+n_{b z} a_{b y}-n_{b y} a_{b z}, \\
& r_{b y}=-n_{y} \cos \xi_{0}+n_{b x} a_{b z}-n_{b z} a_{b x}, \\
& r_{b z}=-n_{z} \cos \xi_{0}+n_{b y} a_{b x}-n_{b x} a_{b y} .
\end{aligned}
$$

In order to calculate the caustic points, all rays reflected from focal surface to the focal region will be expressed and displayed. The focal region consists of a set of focal points, $F_{b}=\left[F_{b x}, F_{b y}, F_{b z}\right]$. The equations of the $F_{b}$ defined in $x-, y$-, and $z$-coordinates are derived as follows:

$$
\begin{aligned}
& F_{b x}=P_{r x}+\rho r_{b x}, \\
& F_{b y}=P_{r y}+\rho r_{b y}, \\
& F_{b z}=P_{r z}+\rho r_{b z} .
\end{aligned}
$$

The focal points are plotted and the most converged area or point is determined as caustic and will be used throughout the study for the calculation of feed position.
3.3. Transverse Plane. The configuration of parabolic antenna with the illustration of transverse plane is shown in Figure 5. From the diagram, it shows that the incident rays exist in a plane perpendicular to the previous scanning plane. The incident rays will cross the $y$-axis before being reflected by the reflector surface; hence, the $y$-coordinates remain unchanged throughout the procedure.

As explained in scanning plane, the reflector points, $P(\theta)$, will be determined in terms of $\rho, \theta$, and $\phi$, where $\phi$ is defined as the azimuth angle measured from the centre of reflector as illustrated in Figure 1 . Thus, the $P(\theta)$ in $x$-, $y$-, and $z$-direction is determined by the following equations:

$$
\begin{gathered}
P_{r x}=\rho \sin \theta \cos \phi, \\
P_{r y}=\rho \sin \theta \sin \phi, \\
P_{r z}=-\rho \cos \theta .
\end{gathered}
$$

However, in this case, the values of $\theta$ and $\phi$ are unknown and will be determined by solving various equations. In transverse plane, $P_{r y}$ is fixed to $y_{1}$, which ranges from $-D / 2$ to $D / 2$. Based on the condition of incident rays in Figure 5 , the following expression is derived:

$$
\tan \theta_{\text {in }}=-\frac{P_{r x}}{P_{r z}}=\frac{\sin \theta_{m} \cos \phi}{\cos \theta_{m}} .
$$

By substituting $\rho$ to the equation of $P_{r y}$ in (14), the following equation is obtained:

$$
\frac{2 f}{1+\cos \theta_{m}} \sin \theta_{m} \sin \phi=y_{1} .
$$

In order to derive the value of $\theta$ and $\phi$, (15) and (16) will be solved simultaneously. By using the technique called "completing the square," the quadratic equations can be solved and hence, the values of $\theta$ and $\phi$ are obtained. Thus, the exact parabolic surface points as mentioned in (14) can be determined. On the other hand, the plane of incident rays, $P_{\text {in }}\left(P_{\text {in } x}, P_{\text {in } y}, P_{\text {in } z}\right)$ is calculated by solving the position of the ray in $z$-direction:

$$
P_{\text {in } z}=P_{r z} \sin ^{2} \theta_{\text {in }}-P_{r y} \sin \theta_{\text {in }} \cos \theta_{\text {in }}+l_{o} \cos \theta_{\text {in }} .
$$


TABLE 1: Study parameters.

\begin{tabular}{lc}
\hline Antenna parameters & Numerical values \\
\hline Frequency $(\mathrm{GHz})$ & $11.4 \mathrm{GHz}(\lambda=26.3 \mathrm{~mm})$ \\
Antenna diameter $(\mathrm{mm})$ & $D=20 \lambda=526.3 \mathrm{~mm}$ \\
& $f_{1}=526 \mathrm{~mm}(f / D=1)$ \\
Focal length $(\mathrm{mm})$ & $f_{2}=789 \mathrm{~mm}(f / D=1.5)$ \\
& $f_{3}=1578 \mathrm{~mm}(f / D=3)$ \\
Incident beam direction $\left({ }^{\circ}\right)$ & $\theta_{\text {in }}=-35^{\circ}$ to $35^{\circ}$ \\
\hline
\end{tabular}

Since the rays are rotated along the $y$-axis, thus, similar to the $P_{r y}$, the incident points also have the same values:

$$
P_{\text {in } y}=P_{r y} .
$$

The calculation of the $P_{\text {in } x}$ is based on the same principle adopted in scanning plane, and thus

$$
P_{\text {in } x}=-\cot \theta_{\text {in }}\left(P_{\text {in } z}-\frac{l_{o}}{\cos \theta_{\text {in }}}\right) .
$$

The reflected vectors are calculated by deriving the normal vectors as in (10) and the prediction of the feed position can be performed by using the same concept as done in scanning plane.

\section{Results and Discussion}

4.1. Parametric Setup. Ray tracing program for scanning and transverse plane is performed for all major points on the reflector. The analysis is carried out for a set of incident angles $\theta_{\text {in }}$ to interpolate the locus of caustic and to observe the caustic behaviour. Critical antenna parameter such as $f / D$ is varied to evaluate the effects towards the caustics. To vary the $f / D$, the reflector diameter is kept constant at $526.3 \mathrm{~mm}$, whilst only the focal length, $f$, is changed. The parameters of study are summarized in Table 1 .

4.2. Displacement of Caustics. The results of focal region ray tracing for scanning plane is shown in Figure 6. In this analysis, the focal region of the parabolic reflector is analyzed for five different incident angles, which are set within the range of $\theta_{\text {in }}=-35^{\circ}$ to $35^{\circ}$. The results are observed in $x z$-plane, a two-dimensional graph that best represents the configuration of the scanning plane mode. Based on the graphs, locus of caustic can be interpolated as the incident direction is varied. The graphs are drawn on the same scale, thus the changes in antenna configuration and caustic behaviour can be demonstrated.

From the results, it can be observed that as the incident beam is increased, the caustics moved further away from the origin. As seen from the zoomed diagrams, the quality of focusing also changed, which means that the caustics are less converged at higher $\theta_{\text {in }}$. On the contrary, the caustics are more converged and easier to determine when the $f / D$ is increased. Therefore, it is expected that at higher $f / D$ configuration the calculation of caustic points is more accurate and thus provide more reliable results. The caustic points for scanning plane are measured in terms of $x$ - and $z$ axis displacement or denoted as $\Delta x_{s}$ and $\Delta z_{s}$, respectively. As seen from Figure $6(\mathrm{c})$, a line is drawn to interpolate the locus of caustic. The line of caustic locus is represented by a variable $S(x, z)$, which represents the distance between the centre of parabolic reflector to the caustic points.

Figure 7 shows the results of transverse plane ray tracing. Due to the complexity of the transverse plane, the behaviours of the transverse plane caustics are best observed in threedimensional axes. The axes are rotated several times during measurement in order to calculate the $x$ - and $z$-axis displacement, also known as $\Delta x_{t}$ and $\Delta z_{t}$, respectively. From the graphs, it can be seen that the caustics are less converged at higher $\theta$ in. However, similar to scanning plane, the effects are less when the $f / D$ is increased. The caustic locus is interpolated and the distance $S(x, z)$ as shown in Figure 7 (c) is calculated and will be used in the next analysis.

4.3. Equation of Caustic Locus. The results of caustic displacements are plotted, and the trend is observed. Specific examples of the caustic points, with the comparison between two different reflector configurations $(f / D=1.5$ and 3$)$, are presented in Figures 8 and 9 for scanning plane and transverse plane, respectively. The convergence of parallel incident rays with $\theta_{\text {in }}=-20^{\circ}$ is shown in both diagrams. The caustics of other incident angles within the range of $\pm 35^{\circ}$ are plotted. In transverse plane, a clearer view of the convergence of rays is shown in the zoomed image.

Based on the results of caustic displacements, the trajectory of the caustics can be approximated as follows:

(a) in the scanning plane

$$
S(x, z)=f \cos \theta_{\text {in }},
$$

(b) in the transverse plane

$$
S(x, z)=\frac{f}{\cos \theta_{\text {in }}} .
$$

Hence, the locus of the caustics in the scanning plane is a circular arc with a curvature that is smaller by $\cos \left(\theta_{\text {in }}\right)$. Meanwhile, for the transverse plane, the trajectory is also a circular arc, but with a curvature that is bigger by $\cos \left(\theta_{\text {in }}\right)$. Thus, if an antenna system is to be designed to form a beam at the desired direction given by $\theta_{\text {in }}$, the feed will be positioned on the curvature given by (20) and (21) on the scanning plane and the transverse plane, respectively. The accuracy and validity of the loci equations can be shown in Figure 10. In this diagram, the normalized caustic displacement data obtained from both scanning plane and transverse plane analysis are plotted and lines joining the points are drawn to interpolate the locus. The lines of equations for caustic loci are also plotted according to the incident beam direction. Both results are compared, and based on the plots, it can be observed that the lines of (20) and (21) almost matched with the caustic displacement data of scanning and transverse plane, respectively. Therefore, the general equations for caustic loci can be used as a guide to preliminary locate the feed positions of parabolic reflector antenna, especially for the design of multibeam or shaped beam system. 


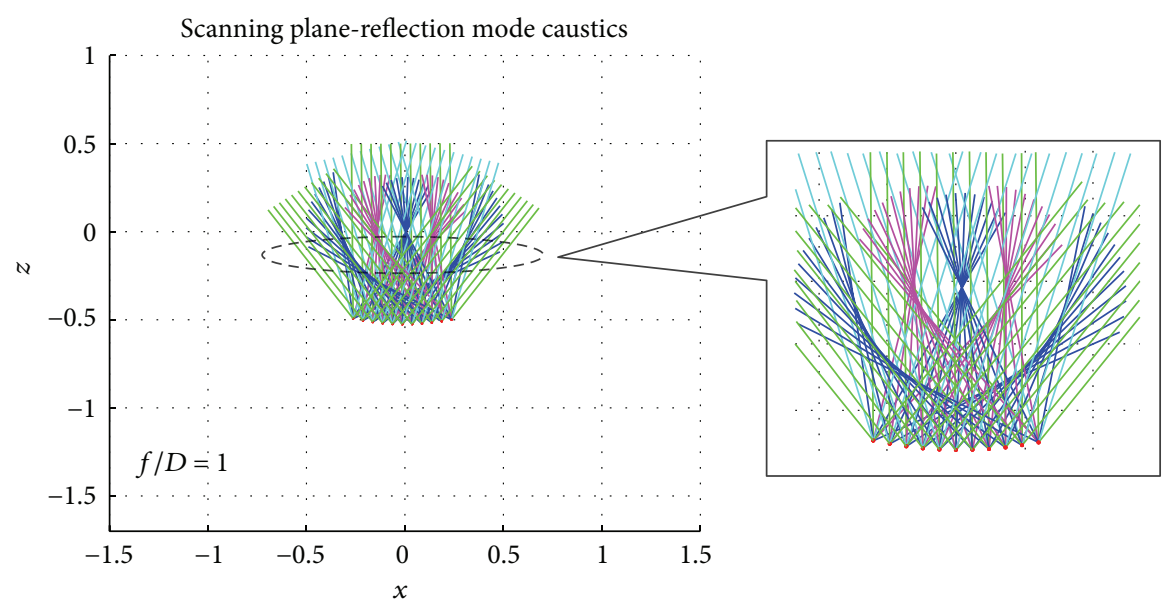

(a)

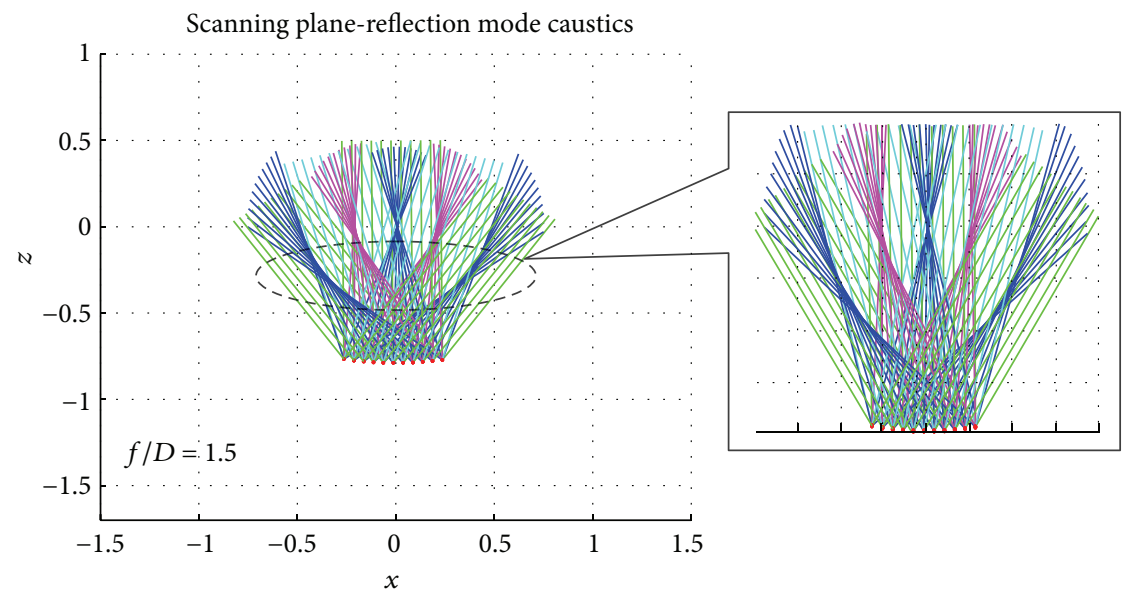

(b)

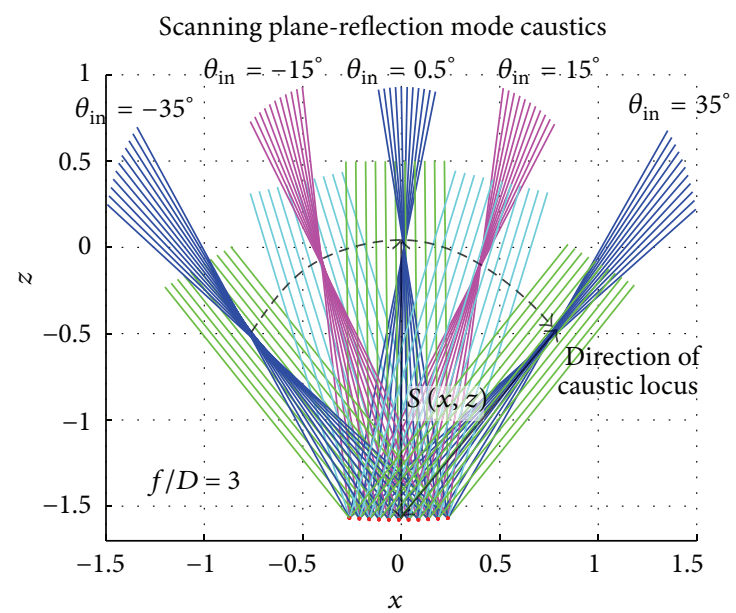

(c)

FIGURE 6: Results of scanning plane ray tracing for various incoming beam directions (a) $f / D=1$, (b) $f / D=1.5$, and (c) $f / D=3$.

4.4. Radiation Characteristics. To ensure the accuracy of the method, the results of caustic obtained in the previous section are verified through the calculation of the radiation pattern. The relative radiation pattern is calculated by using the following equations, where $\xi(x, y)$ represents the aperture phase distribution obtained through 


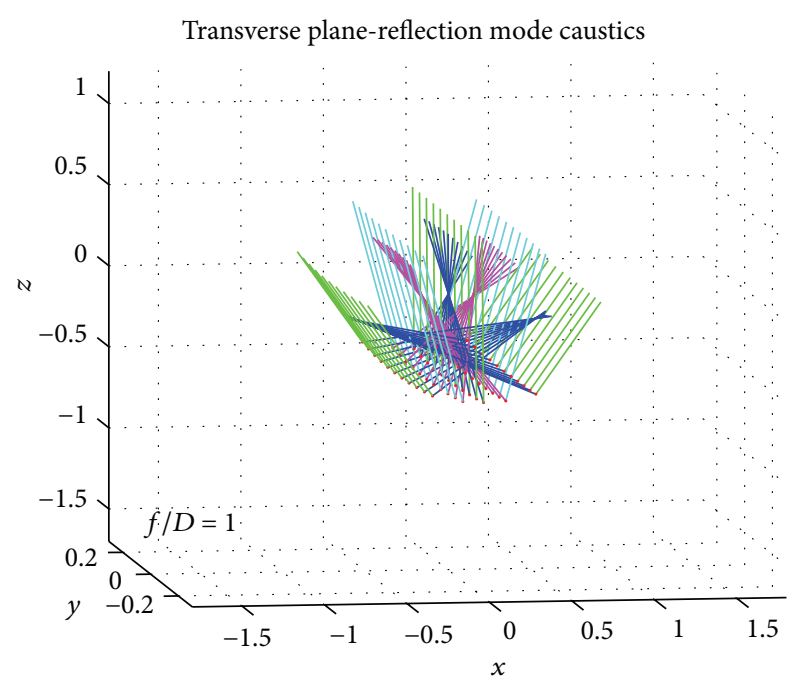

(a)

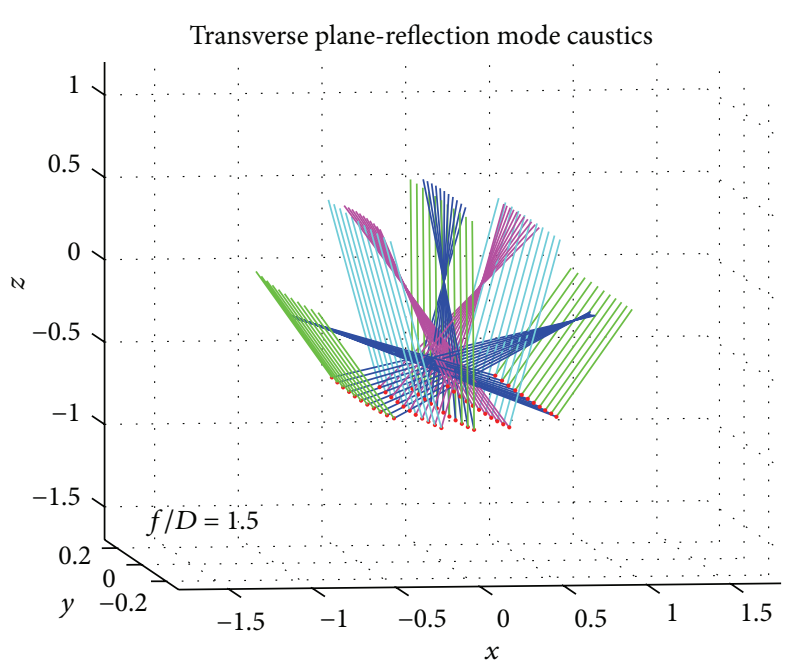

(b)

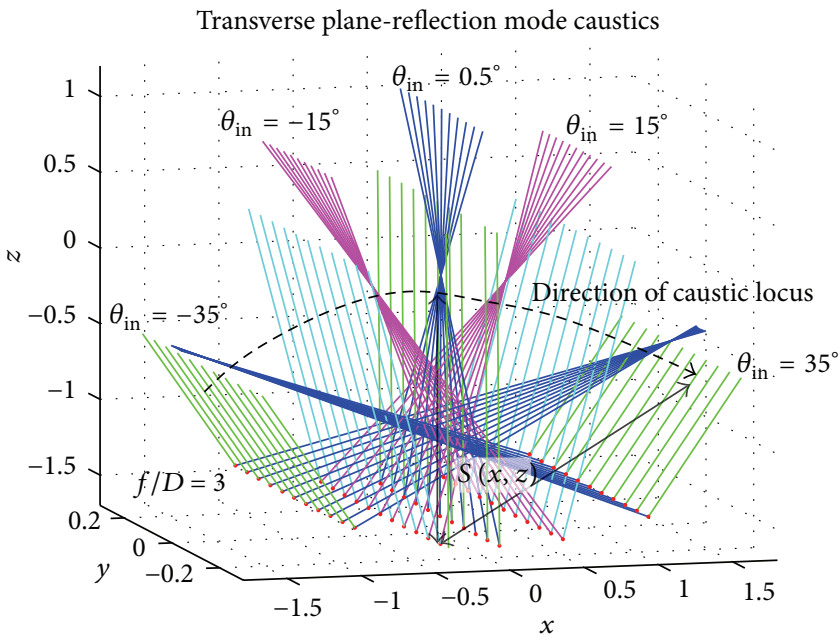

(c)

FiguRe 7: Results of transverse plane ray tracing for various incoming beam directions (a) $f / D=1$, (b) $f / D=1.5$, and (c) $f / D=3$.

comparison of ray length from a feed to all aperture points [11]:

$$
\begin{array}{r}
E_{r}=\int\left\lfloor\int e^{\left(j k r \sin \theta \cos \left(\Phi-\Phi^{\prime}\right)+j \zeta(x, y)\right)} d \Phi^{\prime}\right\rfloor r d r, \\
\text { where } r=\rho \sin \theta .
\end{array}
$$

Several examples based on the incident direction of $\theta_{\text {in }}=$ $-20^{\circ}$ are shown here in order to demonstrate the behaviour of the radiation pattern. Figures 11 and 12 show the radiation characteristics of a parabolic reflector with $f / D=1.5$. In these configurations, the feed locations $F(x, y, z)$ are set to be located at two different positions. Firstly, the feed is set at the caustic point $\left(\Delta x_{s}, \Delta z_{s}\right)$ or $\left(\Delta x_{t}, \Delta z_{t}\right)$ that has been obtained in the previous ray tracing program for scanning plane and transverse plane, respectively. Then, the result is compared to the radiation pattern measured when the feed is placed at the position determined through the locus equation, (20) and (21). The results of both scanning plane and transverse plane are shown.

From Figure 11, the maximum gain is measured at around $20^{\circ}$ offset from the origin, which is the expected result since the feed location was previously set at the $-20^{\circ}$ caustic point. However, the plot given by (20) gives better representation of the exact beam shift. Meanwhile, as for transverse plane, more deteriorate patterns are observed.

For both scanning and transverse planes, by comparing the caustic displacement results with the "blue" line, it seems like the locus equations for both scanning and transverse produced more accurate beams, with the highest gain obtained at the direction closer to the desired beam direction. In this case, the equations of caustic loci become very useful in determining the accurate position of the feed. However, as expected, the analysis conducted in scanning mode provides 


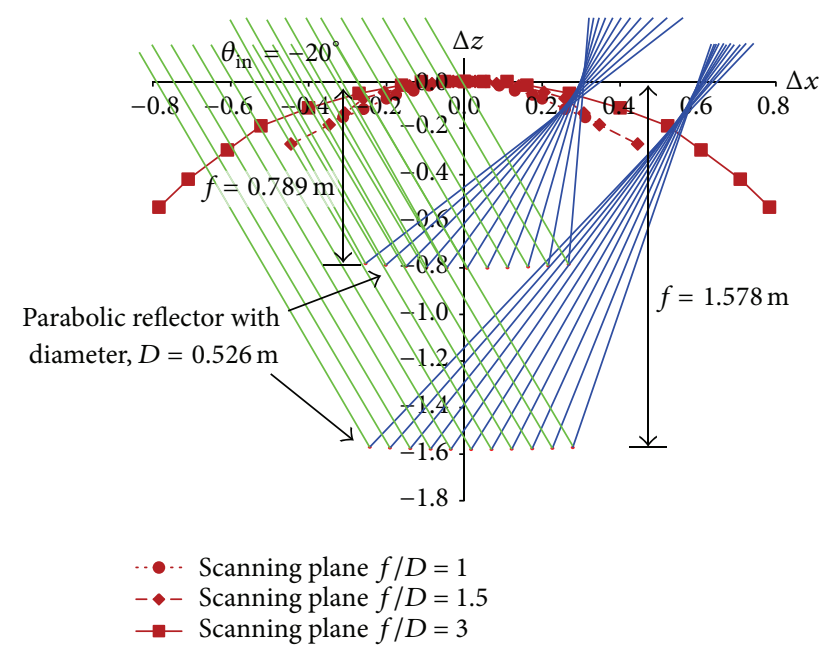

Figure 8: Scanning plane caustic in $x z$-plane, $\theta_{\text {in }}=-20^{\circ}$ with $f / D=1.5$ and 3 .

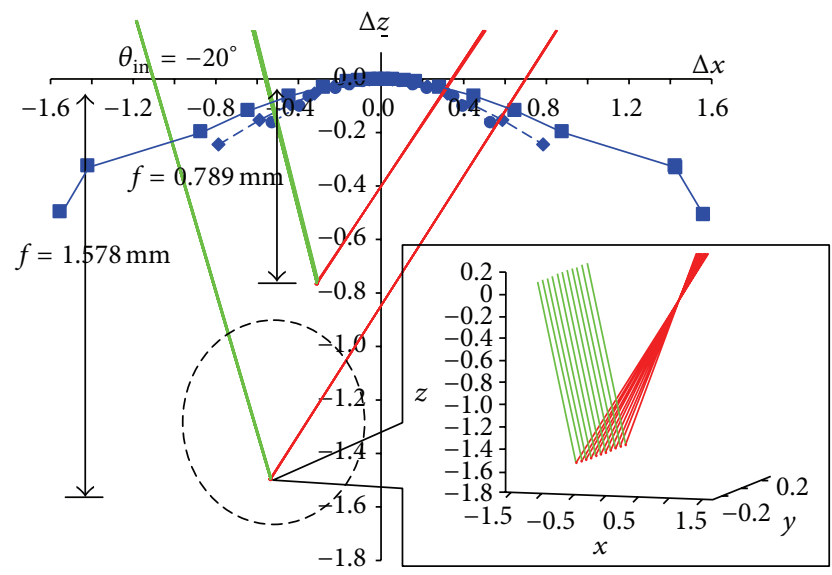

-.. Transverse plane $f / D=1$

- - Transverse plane $f / D=1.5$

$\rightarrow$ Transverse plane $f / D=3$

Figure 9: Transverse plane caustic in $x z$-plane, $\theta_{\text {in }}=-20^{\circ}$ with $f / D=1.5$ and 3 .

more accurate results as compared to the transverse mode. To investigate further, similar exercise is carried out for $f / D=3$ configuration.

Similar to $f / D=1.5$, it can be observed from Figures 13 and 14 that the equations of caustic loci bring the beam direction closer to the desired $\theta_{\text {in }}=-20^{\circ}$ direction although it seems like in transverse plane, the result deviates further as compared to scanning plane. As shown by scanning plane in Figure 13, the radiation patterns for both feed configurations overlapped each other due to the similarity of both values. The highest gain is also measured at the same point. The agreement of the radiation gain values obtained at the two different feed configurations validates the equation of caustic loci derived by author in the previous section. Therefore, the accuracy of the ray tracing algorithms for scanning plane and transverse plane is ensured.

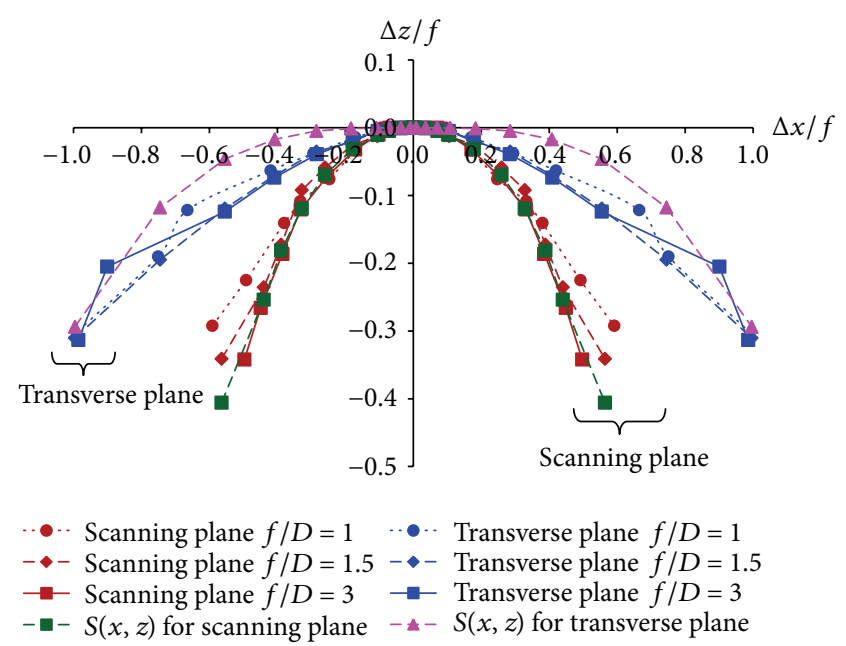

Figure 10: Comparison of two-dimensional caustic displacement with the equation lines of caustic loci, $S(x, z)$.

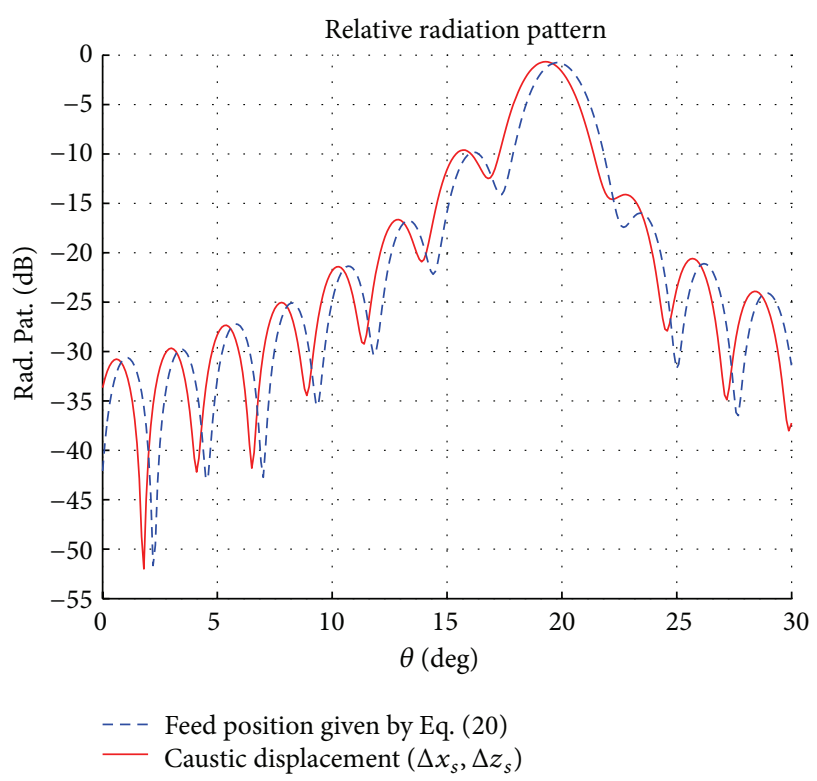

FIGURE 11: Comparison of relative radiation patterns using scanning plane caustic and locus for $f / D=1.5$ configuration.

\section{Conclusion}

A set of algorithms developed for the scanning plane and transverse plane ray tracing program are presented. The results obtained from the focal region ray tracing are discussed, and based on the caustic displacement results, useful equations representing the caustic loci are derived. The importance of the equations are shown, which could be used in designing a multibeam antenna application. Off-feed caustic displacements obtained in ray tracing method are compared with the approximate equation of locus through calculation of radiation pattern. The results of beam shift show good agreement between these two values, and thus, the accuracy of the ray tracing method and the reliability of 


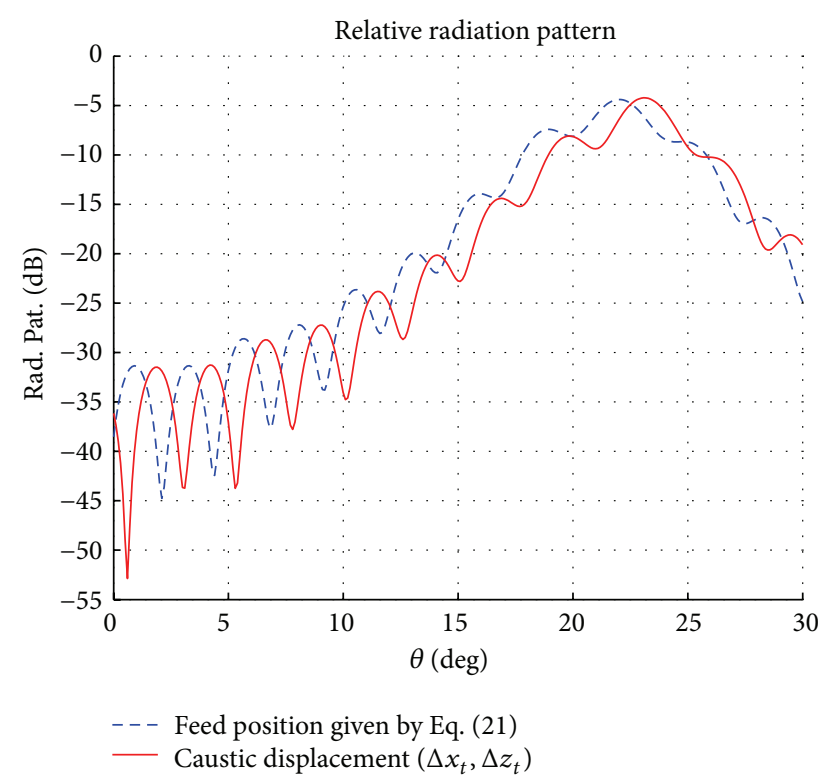

Figure 12: Comparison of relative radiation patterns using transverse plane caustic and locus for $f / D=1.5$ configuration.

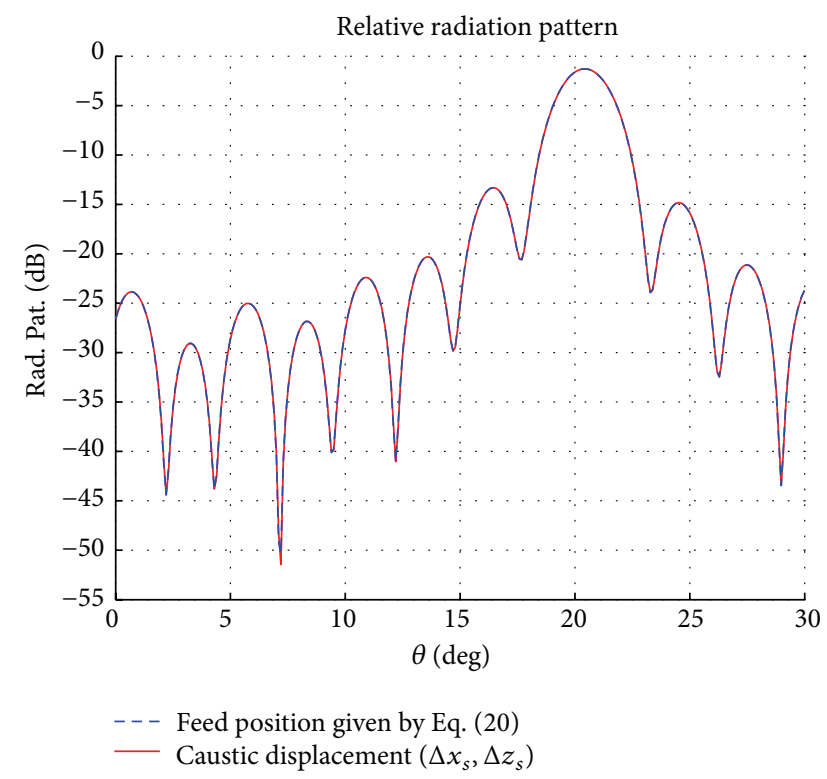

FIGURE 13: Comparison of relative radiation patterns using scanning plane caustic and locus for $f / D=3$ configuration.

the locus equation for scanning plane and transverse plane analysis are proven.

\section{Conflict of Interests}

The authors declare that there is no conflict of interests regarding the publication of this paper.

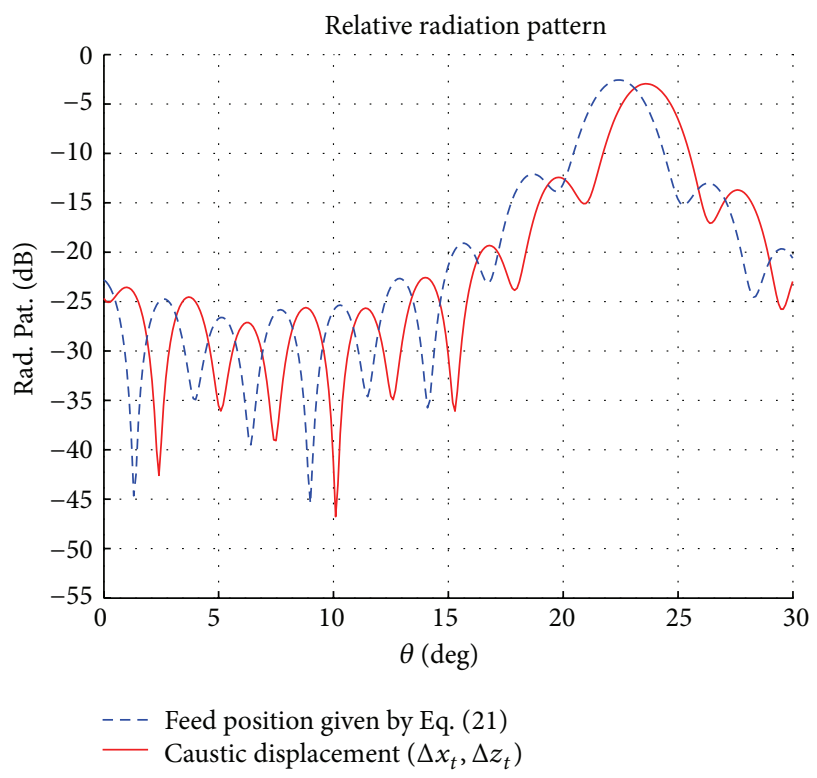

FIGURE 14: Comparison of relative radiation patterns using transverse plane caustic and locus for $f / D=3$ configuration.

\section{Acknowledgment}

The authors would like to thank Universiti Teknologi MARA for continuous support towards this research project.

\section{References}

[1] R. Azim, M. T. Islam, N. Misran, S. W. Cheung, and Y. Yamada, "Planar UWB antenna with multi-slotted ground plane," Microwave and Optical Technology Letters, vol. 53, no. 5, pp. 966-968, 2011.

[2] L. Liu, S. W. Cheung, R. Azim, and M. T. Islam, "A compact circular-ring antenna for ultra-wideband applications," Microwave and Optical Technology Letters, vol. 53, no. 10, pp. 2283-2288, 2011.

[3] K. Shogen, S. Tanaka, and S. Nakazawa, "Studies on the onboard Antennas for $21 \mathrm{GHz}$ band broadcasting satellites and future study items," IEICE Transactions on Communications, vol. J94B, no. 9, pp. 1014-1024, 2011.

[4] G. Toso, C. Mangenot, and P. Angeletti, "Recent advances on space multibeam antennas based on a single aperture," in Proceedings of the European Conference on Antennas and Propagation, pp. 454-458, April 2013.

[5] S. Yun, M. Uhm, J. Choi, and I. Yom, "Multibeam reflector antenna fed by few elements for Ka-band communication satellite," in Proceedings of the IEEE Antennas and Propagation Society International Symposium, pp. 1-2, July 2012.

[6] M. Schneider, C. Hartwanger, and H. Wolf, "Antennas for multiple spot beam satellites," CEAS Space Journal, vol. 2, no. 1-4, pp. 59-66, 2011.

[7] M. Mahajan, R. Joti, K. Sood, and S. B. Sharman, "A method of generating simultaneous contoured and pencil beams from single shaped reflector antenna," IEEE Transactions on Antennas and Propagation, vol. 61, no. 10, pp. 5297-5301, 2013.

[8] R. E. Collin and F. J. Zucker, Antenna Theory: Part 2, McGrawHill, New York, NY, USA, 1969. 
[9] C. J. Sletten and R. A. Shore, "Focal Surfaces of offset dualreflector antennas," IEE Proceedings H: Microwaves Optics and Antennas, vol. 129, no. 3, pp. 109-115, 1982.

[10] Y. Yamada and S. Sasaki, "Estimations of radiation characteristics of an Off-focus feed shaped dielectric lens antenna by a ray tracing method," in Proceedings of the IEEE Antennas and Propagation Society International Symposium, pp. 398-401, San Antonio, Tex, USA, June 2002.

[11] C. A. Balanis, Antenna Theory: Analysis and Design, John Wiley and Sons, New York, NY, USA, 3rd edition, 2005.

[12] W. L. Stutzman and G. A. Thiele, Antenna Theory and Design, John Wiley and Sons, New York, NY, USA, 2nd edition, 1998.

[13] N. Abd Rahman, M. Islam, N. Misran, Y. Yamada, and N. Michishita, "Design of a aatellite antenna for Malaysia beams by ray tracing method," in Proceedings of the International Symposium on Antennas and Propagation (ISAP '12), pp. 13851388, October 2012. 

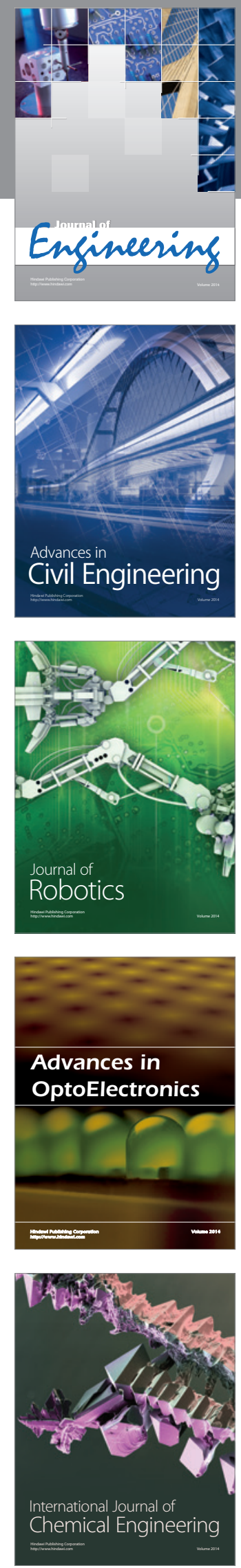

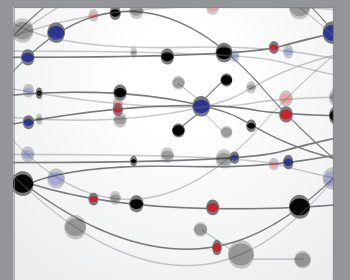

The Scientific World Journal
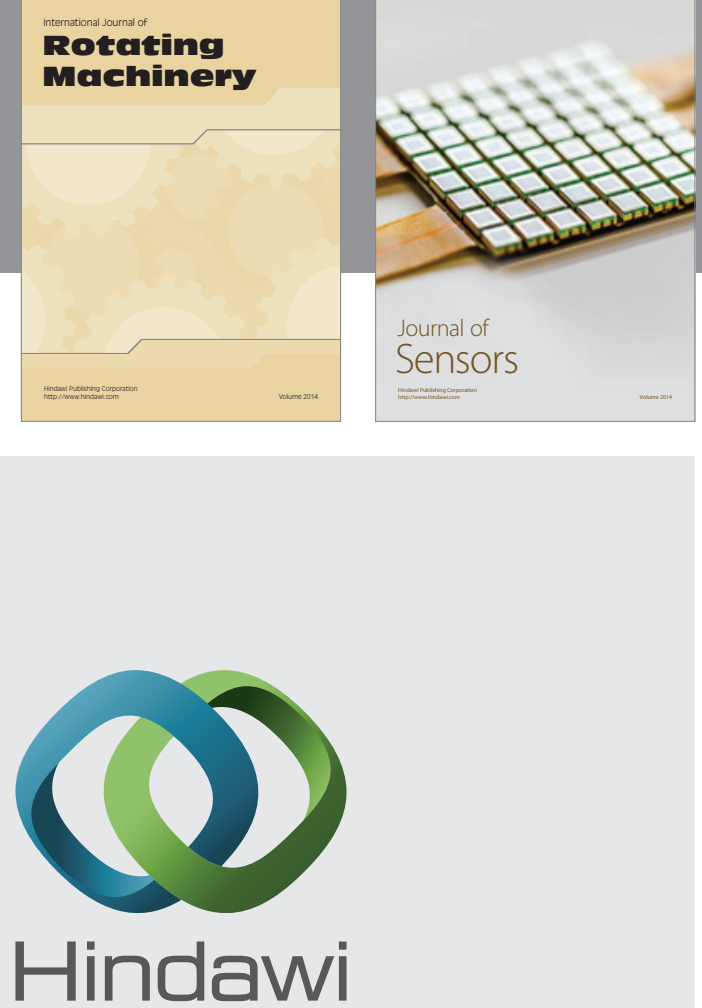

Submit your manuscripts at http://www.hindawi.com
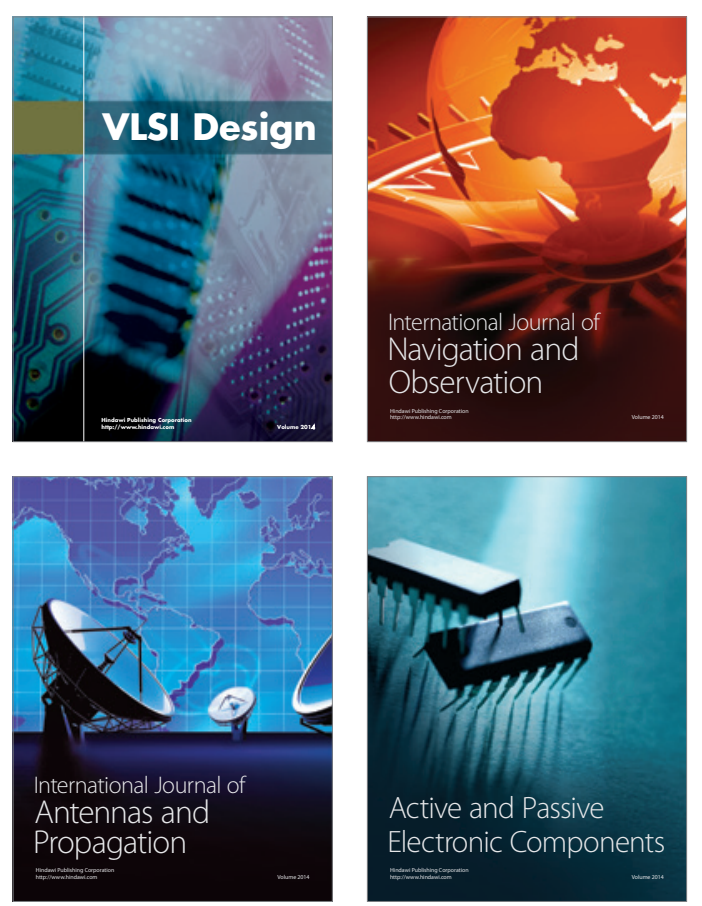
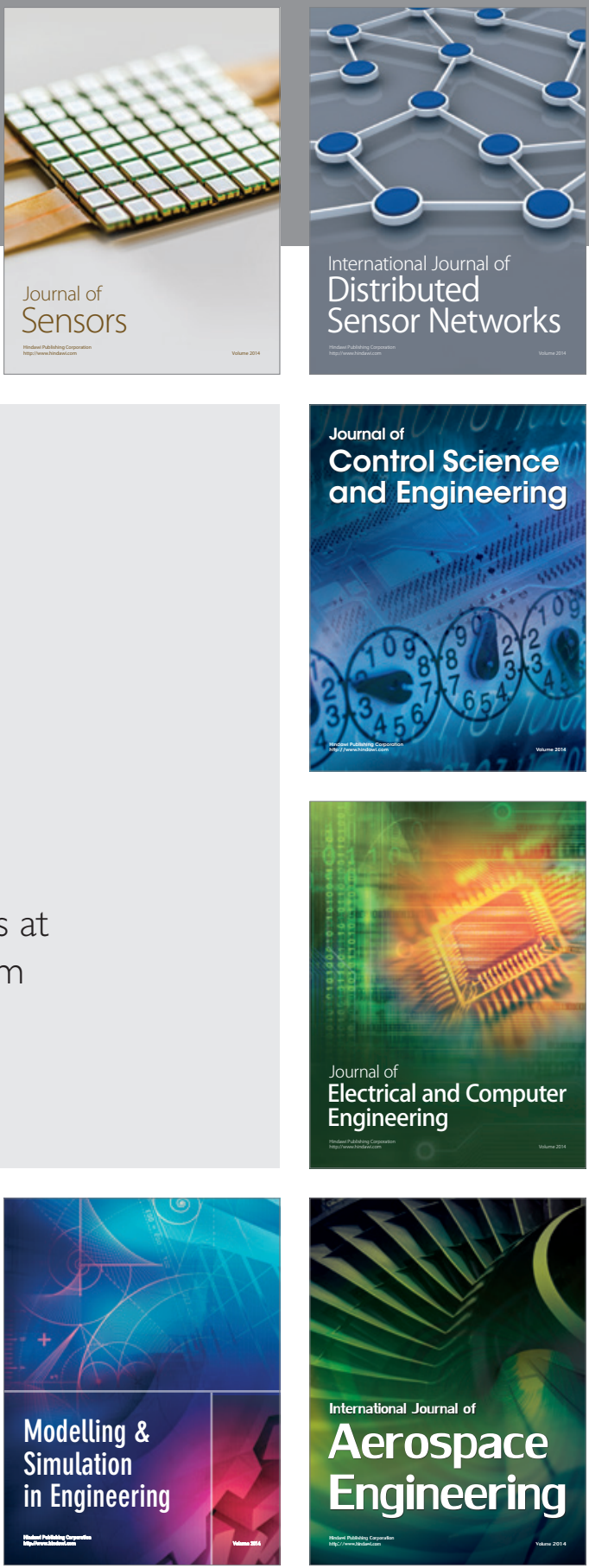

Journal of

Control Science

and Engineering
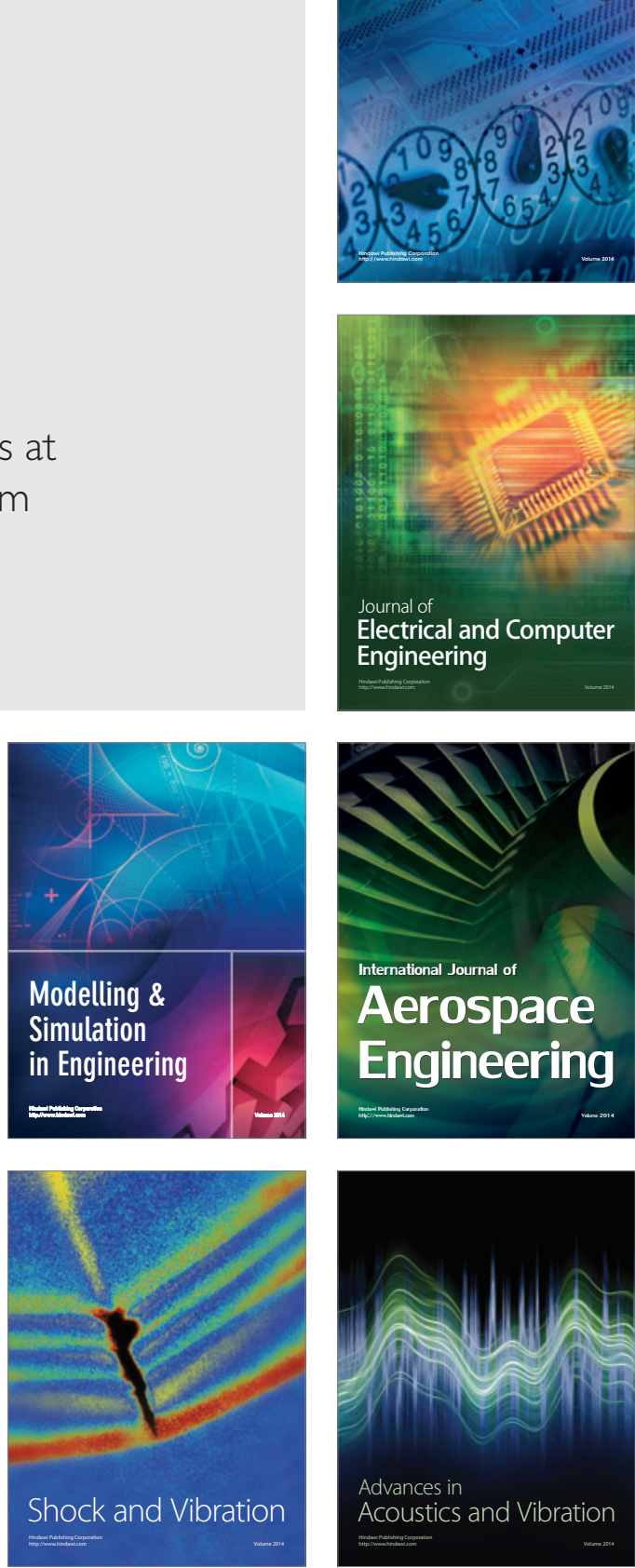